\title{
The Role of Microtubule Detyrosination in Axon Regeneration
}

\author{
Philipp Gobrecht, Daniel Terheyden-Keighley and Dietmar Fischer* \\ Department of Cell Physiology, Faculty of Biology and Biotechnology, Germany
}

*Corresponding author: Dietmar Fischer, Department of Cell Physiology, Faculty of Biology and Biotechnology, Bochum, Germany

\begin{tabular}{ll} 
ARTICLE INFO & ABSTRACT \\
\hline
\end{tabular}

Received: 蔧 April 02, 2019

Published: 幽 April 10, 2019

Citation: Philipp G, D Terheyden-Keighley, D Fischer. The Role of Microtubule Detyrosination in Axon Regeneration. Biomed J Sci \& Tech Res 17(1)-2019. BJSTR. MS.ID.002932.
Keywords: Microtubule Detyrosination; Parthenolide; Gsk3; Map1b; Parthenolide; Drg Neuron; Axon Regeneration; Peripheral Nerve Regeneration

\section{Opinion}

Axonal lesions of the peripheral nervous system (PNS) disconnect their targets from the central nervous system (CNS), resulting in motoric and sensory impairments. While axons of the PNS are generally able to regrow, functional recovery often remains incomplete. This is because, under normal conditions, axons regenerate with a speed of 1-2 mm per day [1,2]. This speed, however, is only kept up for a few months, after which neurons turn off their intrinsic growth program and Schwann cells lose their growth promoting functions [1,3]. This timeframe enables axons of the PNS to regenerate a distance of approximately $9-18 \mathrm{~cm}$, which is not sufficient for many nerve injuries that can span distances of up to one meter. Axons that are unable to reinnervate their original targets do not just leave patients with functional deficits but are also often misguided and form improper innervations known as neuromas. These cause chronic pain that is difficult to treat. Consequently, it has been shown that accelerating peripheral axon growth improves the general outcome of the motor and sensory recovery after nerve injury [4]. However, despite years of research, no treatments that accelerate the axon growth rate are clinically available [5].

One crucial determinant of axon growth speed is the dynamics of microtubule filaments (MTs). MTs are part of the neuronal cytoskeleton that maintains axonal polarity. Their cylinder-like structure with a diameter of $25 \mathrm{~nm}$ is made up of 13 heterodimers consisting of $\alpha$ and $\beta$ tubulins. In axons, MTs are mainly organized with the $\alpha$ tubulin facing towards the axonal tip (the so-called "+ end"), and the $\beta$ tubulin is facing towards the soma (the socalled "- end"). In the tip of extending axons, the growth cone, more tubulin heterodimers are added to the MT "+ end" than removed, thus pushing the growth cone forward. The speed of addition of new heterodimers to the "+ end" is, among other factors, dependent on various posttranslational modifications of the $\alpha$ tubulin [6]. One such posttranslational modification is the removal of the C-terminal tyrosine residue [7]. This detyrosination is mediated by a so far unknown tubulin carboxypeptidase. Since mainly tyrosinated tubulin heterodimers are added to the "+ tips" of MTs, and these restrict the rate of MT polymerization, growth cone progression speed is controlled by modifying the tyrosination status of $\alpha$ tubulins. Thus, decreasing MT detyrosination could be a potential method for accelerating axon regeneration and consequently functional recovery after PNS injury. Consistent with this hypothesis, the addition of tyrosinated tubulin heterodimers to MTs occurs significantly faster in cultured cell lines than detyrosinated ones [8]. Moreover, several studies demonstrated a correlation between glycogen synthase kinase 3 (GSK3) activityinduced phosphorylation of microtubule-associated protein $1 \mathrm{~B}$ (MAP1B) and reduction of microtubule detyrosination as well as acceleration of axon extension of embryonic PNS neurons [9-12].

Although the molecular mechanism directly linking MAP1B phosphorylation and MT detyrosination has not yet been established, it is generally assumed that phospho-MAP1B interacts 
with the tubulin-carboxypeptidase or a tubulin-tyrosine to reduce MT detyrosination, thus improving axon growth [9,10,13-15]. In agreement with these ideas, we recently demonstrated that similar mechanisms are also relevant for regenerative processes of axons in the adult PNS. Constitutively active GSK3 reduced MT detyrosination and subsequently increased axon growth in cultured sensory neurons almost two-fold [16,17]. We also demonstrated that the GSK3-induced MT detyrosination was, in fact, responsible for the increased axon regeneration as pharmacological inhibition of MT detyrosination with parthenolide, a sesquiterpene lactone that naturally occurs in the plant feverfew (Tanacetum parthenium) also decreased MT detyrosination in the axon tips and fully mimicked the axon growth-promoting effect in cultured PNS neurons [16]. Strikingly, constitutively active GSKe and parthenolide also markedly accelerated axonal regeneration in living animals $[16,17]$. A single injection of parthenolide into the lesioned sciatic nerve or the systemic application of the drug was sufficient to significantly increase the number and length of regenerating axons in the distal nerve three days post-lesion [16].

Also, neuromuscular junctions were re-associated with axons already four days after nerve crush, while none were detectable in vehicle-treated animals. Consequently, parthenolide treatment markedly accelerated the recovery and almost halved the time to restore motor and sensory function of injured animals. These effects were achieved at very low doses, and the animals did not show any signs of side effects [16]. The efficacy of systemically applied parthenolide for the therapeutic promotion of nerve regeneration is particularly promising as recurrent treatments are facilitated compared to local invasive nerve injections. It remains, however, to be seen if a continued systemic parthenolide application can even further accelerate and improve functional recovery also in other species and animal models lacking the ability to fully restore function after a nerve injury, for example after complete nerve transection. These studies are underway. Therefore, we consider drugs, such as parthenolide that inhibit detyrosination of microtubules and thereby increase their dynamics in axonal growth cones to be attractive candidates for further validation as therapeutic agents for traumatic nerve injury. In addition, it seems feasible that these drugs may also be beneficial for disease and drug-induced generalized neuropathies multifocal axonal damage, which severely limits the quality of life of more and more affected patients.

\section{References}

1. Sulaiman W, Gordon T (2013) Neurobiology of peripheral nerve injury, regeneration, and functional recovery: from bench top research to bedside application. The Ochsner journal 13: 100-108.
2. Sunderland S (1947) Rate of regeneration in human peripheral nerves; analysis of the interval between injury and onset of recovery. Arch Neurol Psychiatry 58: 251-295.

3. Hoke A (2006) Mechanisms of Disease: what factors limit the success of peripheral nerve regeneration in humans? Nature clinical practice Neurology 2: 448-454.

4. Ma CH, Omura T, Cobos EJ, Latremoliere A, Ghasemlou N, et al. (2011) Accelerating axonal growth promotes motor recovery after peripheral nerve injury in mice. J Clin Invest 121: 4332-4347.

5. Diekmann H, Fischer D (2016) Parthenolide: a novel pharmacological approach to promote nerve regeneration. Neural Regen Res 11: 15661567.

6. Fukushima N, Furuta D, Hidaka Y, Moriyama R, Tsujiuchi T, et al. (2009) Post-translational modifications of tubulin in the nervous system. J Neurochem 109: 683-693.

7. Hallak ME, Rodriguez JA, Barra HS, Caputto R (1977) Release of tyrosine from tyrosinated tubulin. Some common factors that affect this process and the assembly of tubulin. FEBS letters 73: 147-150.

8. Kreis TE (1987) Microtubules containing detyrosinated tubulin are less dynamic. The EMBO journal 6: 2597-2606.

9. Goold RG, Owen R, Gordon-Weeks PR (1999) Glycogen synthase kinase 3 beta phosphorylation of microtubule-associated protein $1 \mathrm{~B}$ regulates the stability of microtubules in growth cones. J Cell Sci 112: 3373-3384.

10. Lucas FR, Goold RG, Gordon-Weeks PR, Salinas PC (1998) Inhibition of GSK-3beta leading to the loss of phosphorylated MAP-1B is an early event in axonal remodelling induced by WNT-7a or lithium. J Cell Sci 111: 1351-1361.

11. Owen R, Gordon-Weeks PR (2003) Inhibition of glycogen synthase kinase 3beta in sensory neurons in culture alters filopodia dynamics and microtubule distribution in growth cones. Mol Cell Neurosci 23: 626-637.

12. Barnat M, Benassy MN, Vincensini L, Soares S, Fassier C, et al. (2016) The GSK3-MAP1B pathway controls neurite branching and microtubule dynamics. Mol Cell Neurosci 72: 9-21.

13. Gonzalez-Billault C, Engelke M, Jimenez-Mateos EM, Wandosell F, Caceres A, et al. (2002) Participation of structural microtubule-associated proteins (MAPs) in the development of neuronal polarity. J Neurosci Res 67: 713-719.

14. Gonzalez-Billault C, Jimenez-Mateos EM, Caceres A, Diaz-Nido J, Wandosell F, et al. (2004) Microtubule-associated protein 1B function during normal development, regeneration, and pathological conditions in the nervous system. J Neurobiol 58: 48-59.

15. Gobrecht P, Andreadaki A, Diekmann H, Heskamp A, Leibinger M, et al (2016) Promotion of Functional Nerve Regeneration by Inhibition of Microtubule Detyrosination. J Neurosci 36: 3890-3902.

16. Gobrecht P, Leibinger M, Andreadaki A, Fischer D (2014) Sustained GSK3 activity markedly facilitates nerve regeneration. Nature communications 5: 4561 .

17. Gobrecht P, Leibinger M, Andreadaki A, Fischer D (2014) Sustained GSK3 activity markedly facilitates nerve regeneration. Nature communications 5: 4561 . 
ISSN: 2574-1241

DOI: 10.26717/BISTR.2019.17.002932

Dietmar Fischer. Biomed J Sci \& Tech Res

(c) (i) This work is licensed under Creative

Submission Link: https://biomedres.us/submit-manuscript.php

\begin{tabular}{ll}
\hline RIOMEDICAL & Assets of Publishing with us \\
RESARCHES & - Global archiving of articles \\
- Immediate, unrestricted online access \\
- Rigorous Peer Review Process
\end{tabular}

